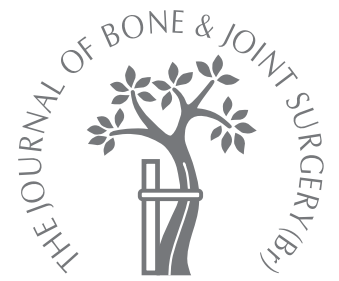

D. G. Campbell, W. W. Duncan, M. Ashworth, A. Mintz, J. Stirling, L. Wakefield, T. M. Stevenson

From The Queen Elizabeth Hospital, Woodville, Adelaide, South Australia

\title{
Patellar resurfacing in total knee replacement
}

\author{
A TEN-YEAR RANDOMISED PROSPECTIVE TRIAL
}

\begin{abstract}
A series of 100 consecutive osteoarthritic patients was randomised to undergo total knee replacement using a Miller-Galante II prosthesis, with or without a cemented polyethylene patellar component. Knee function was evaluated using the American Knee Society score, Western Ontario and McMaster University Osteoarthritis index, specific patellofemoralrelated questions and radiographic evaluation until the fourth post-operative year, then via questionnaire until ten years post-operatively. A ten-point difference in the American Knee Society score between the two groups was considered a significant change in knee performance, with $\alpha$ and $\beta$ levels of 0.05 .

The mean age of the patients in the resurfaced group was 71 years (53 to 88 ) and in the non-resurfaced group was 73 years (54 to 86).
\end{abstract}

After ten years 22 patients had died, seven were suffering from dementia, three declined further participation and ten were lost to follow-up. Two patients in the non-resurfaced group subsequently had their patellae resurfaced. In the resurfaced group one patient had an arthroscopic lateral release. There was no significant difference between the two treatment groups: both had a similar deterioration of scores with time, and no further patellofemoral complications were observed in either group.

We are unable to recommend routine patellar resurfacing in osteoarthritic patients undergoing total knee replacement on the basis of our findings.

Whether to resurface the patella during total knee replacement (TKR) remains controversial. ${ }^{1,2}$ There are proponents for routine patellar resurfacing, for not resurfacing and for selective resurfacing. Some studies suggest that anterior knee pain remains a complication of TKR, with residual patellofemoral pain being present in between $5 \%$ and $45 \%$ of patients. ${ }^{1-10}$

The aetiology of anterior knee pain after replacement is unproven but is generally thought to be related to the patellofemoral joint. Routine patellar resurfacing appears to be an option to reduce patellofemoral-related pain, but prospective randomised trials have not provided consistent results in the short- to medium-term. ${ }^{6,11-16}$ Residual anterior knee pain after TKR is a common cause of early revision surgery, but resurfacing the patella in these circumstances may not relieve the symptoms. ${ }^{17,18}$ Whether the patella is resurfaced appears to depend as much on the surgeon's experience as on the interpretation of data derived from studies often not specifically designed to resolve this question in a scientifically-controlled manner. The Australian Orthopaedic Association National Joint Replace- ment Registry, in its 2004 annual report, ${ }^{19}$ recorded that $57.6 \%$ of 56314 knee replacements were implanted without patellar replacement.

We undertook a controlled clinical trial of patellar resurfacing in osteoarthritic patients treated by TKR. The aim was to produce evidence-based indications for patellar resurfacing in knee replacement. Our hypothesis was that patellar resurfacing would influence the disease-specific outcome of osteoarthritic patients undergoing knee replacement.

\section{Patients and Methods}

A randomised prospective double-blinded control study was conducted using predetermined outcome measures of knee replacement with and without patellar resurfacing. The study was designed with the intention of having a ten-point difference in the 100-point clinical American Knee Society score ${ }^{20}$ between the two groups as the end-point of statistical measurement. In order to determine a ten-point difference in the outcome score with an $\alpha$ (false positive) and a $\beta$ (false negative) level of 0.05 , 37 patients were required in each group, 
assuming a standard deviation of 15 points. A target of 50 patients in each group was chosen to allow for patient loss during the period of study.

Between 1991 and 1993, 100 consecutive patients at The Queen Elizabeth Hospital, Adelaide, were enrolled in a study approved by the Ethical Committee. The indication for surgery was degenerative osteoarthritis, with symptoms sufficient to warrant total knee replacement after the failure of conservative treatment. Patients were excluded from the study if they lived in a remote area, had undergone a previous osteotomy or patellofemoral procedure, had inflammatory arthritis or isolated patellofemoral disease, a varus or valgus deformity of more than $25^{\circ}$ or if there was major bone deficiency on the pre-operative radiographs or at surgery.

The patients were assessed pre-operatively by an independent observer (DGC) and assigned to one of the two treatment groups determined by random number generation. No patient had their treatment group altered at the time of surgery regardless of the patellar findings. The patients were not informed of the surgical treatment to their patellofemoral joint. The clinical assessors (JS and LW) were blinded to the treatment group to which the patient had been allocated. Assessment was conducted independently of routine clinical review.

All patients underwent a Miller-Galante II (Zimmer, Warsaw, Indiana) TKR using prostheses with or without a Miller-Galante II polyethylene patellar component. The posterior cruciate ligament was retained in all cases. Patellar osteophytes were resected when present. The femoral component was externally rotated $3^{\circ}$ from the posterior condylar axis using the guides provided by the manufacturer. Soft-tissue releases were performed as necessary to ensure anatomical tracking of the patella within the patellofemoral groove, without the application of any external stabilising force. The femoral and tibial components were not cemented, with the exception of one tibial component in a patient with markedly osteoporotic bone. All operations were performed or supervised by an experienced consultant surgeon (CB, PD, GM, AM, PL, AMi or TS). Intra-operative observation of the patellar articular cartilage was graded according to the criteria of Outerbridge. ${ }^{21}$ The patellar thickness before and after resurfacing was measured. An assessment of patellar tracking before and, if necessary, after a lateral release was recorded.

Patients were reviewed at a dedicated independent follow-up clinic at three months, six months, one, two and four years post-operatively. They were reviewed further by postal questionnaire at eight and ten years post-operatively. Knee function before and after surgery was evaluated by a disease-specific score (American Knee Society Score) ${ }^{20}$ up to four years post-operatively and by patella-specific questions at all intervals. Questions included the presence of anterior knee pain, the relationship of pain with stair climbing and arising from a chair, and the presence of patellar crepitus. We added the Western Ontario and McMaster
Table I. Demographics of the patients enrolled in the study

\begin{tabular}{lll}
\hline & Resurfaced & Not resurfaced \\
\hline Number & 46 & 54 \\
Mean age in yrs (range) & 71 (53 to 88) & 73 (54 to 86) \\
Gender (F:M) & $33: 13$ & $39: 15$ \\
Side (R:L) & $24: 22$ & $28: 26$ \\
Mean weight (kg) & 88.4 & 77.5 \\
Mean BMI* & 31 & 30.7 \\
Charnley grade & & \\
$\quad$ A & 24 & 28 \\
B & 18 & 22 \\
C & 4 & 4
\end{tabular}

* BMI, body mass index

Table II. Intra-operative grading of the patellar articular surface. Fibrillated cartilage includes Outerbridge ${ }^{21}$ grade 2 and grade 3

\begin{tabular}{|c|c|c|c|c|}
\hline & \multicolumn{2}{|c|}{ Resurfaced } & \multicolumn{2}{|c|}{ Not resurfaced } \\
\hline & Medial & Lateral & Medial & Lateral \\
\hline Normal & 3 & 2 & 4 & 4 \\
\hline Fibrillated & 31 & 35 & 38 & 36 \\
\hline Exposed & 12 & 9 & 12 & 14 \\
\hline
\end{tabular}

University Osteoarthritis Index (WOMAC) 22 for the eightand ten-year postal reviews.

The radiographic evaluation included an assessment of coronal and sagittal knee alignment before and after the replacement, the position of the joint line, patellar altitude as described by Figgie et $\mathrm{al}^{23}$ and the patellar ligament height ratio as described by Insall and Salvati. ${ }^{24}$ Patellar tracking was assessed by a $30^{\circ}$ and a $45^{\circ}$ skyline view. Patellar position was measured as described by Gomes, Bechtold and Gustilo ${ }^{25}$ and graded as central, medial tilt, lateral tilt (more than $5^{\circ}$ ), or laterally displaced if more than $5 \mathrm{~mm}$ from the centre of the trochlear groove. Interface lucencies were described according to the Knee Society Total Knee Arthroplasty roentgenographic evaluation and scoring system. ${ }^{26}$ The competence of the medial retinacular closure was assessed by the addition of two wire sutures placed one at either side of the medial arthrotomy, and the distance between the two was assessed using the width of the patella as a reference.

Data were maintained and analysed on Microsoft Access and Excel software (Microsoft, Redmond, Washington). Student's $t$-test was used for the comparison of univariate means, analysis of variance (ANOVA) was used to compare means of multiple independent groups, and the chi-squared test was used to compare categorical variables. Statistical significance was accepted with $\mathrm{p}$ values $\leq 0.05$.

\section{Results}

The patient demographics are given in Table I. A total of 100 TKRs were performed in 100 patients. The demographics were similar in the two groups, except for a larger 
number of non-resurfaced patellae produced by the randomisation process. The patients who had resurfacing of the patella were a mean of $10.9 \mathrm{~kg}$ heavier but had a comparable body mass index. There was a comparable distribution of Charnley grade A, B and C patients in each group. The intra-operative findings demonstrated similar patellar articular surface gradings (Table II). A lateral release was performed in $29(54 \%)$ of the non-resurfaced group and in $26(57 \%)$ of the resurfaced group. The patellar thickness was accurately restored in the knees which had been resurfaced (mean $-0.14 \mathrm{~mm} ;-3$ to $+2 \mathrm{~mm}$ change in thickness).

At the four-year review, two patients (both not resurfaced) were lost to follow-up, three patients (two not resurfaced, one resurfaced) had developed severe dementia and could not be constructively questioned and 12 patients had died (ten not resurfaced, two resurfaced). Of these, one death was related to a patellofemoral revision procedure in a patient who had undergone patellar resurfacing at the primary procedure, whilst the remaining 11 were unrelated to the replacement surgery. At ten years post-operatively, a total of 22 patients had died, seven had dementia, ten were lost to follow-up and three patients refused to participate in the ten-year postal survey, citing poor general health as the reason. The ten-year review included 28 patients without resurfaced patellae and 30 patients who had undergone patellar resurfacing.

Non-patellofemoral complications. In the group without patellar resurfacing there were two superficial infections, which resolved without surgical intervention. One patient had a fracture of the posteromedial corner of the tibial base plate and required a revision. A further patient had a fracture of the medial tibial plateau which required internal fixation and tibial component revision. Four patients required medical therapy for a deep-vein thrombosis. A prominent retinacular wire marker was removed under local anaesthetic in one patient.

In the patellar resurfaced group, there were three superficial infections, which resolved without surgical intervention. Two patients had significant arthrofibrosis, which required a manipulation in one patient and an arthroscopic synovectomy in the other. One patient had significant polyethylene-related synovitis which resolved following exchange of the tibial polyethylene liner. Two patients required medical therapy for a deep-vein thrombosis and one further patient had a pulmonary embolus. A prominent retinacular wire marker was removed under local anaesthetic in one patient.

Patellofemoral reoperation. In the non-resurfaced group, two patients had their patellae resurfaced because of anterior knee pain. A 60-year-old man with grade II patellar chondromalacia pre-operatively had a patellar resurfacing one year post-operatively. His pain resolved and his Knee Society score ${ }^{20}$ was 160 points. A 60 -year-old woman with normal patellar articular surfaces pre-operatively underwent secondary resurfacing at three years post-operatively because of anterior knee pain. It was observed that her
Table III. Knee Society function scores ${ }^{20}$

\begin{tabular}{llll}
\hline & Resurfaced & Not resurfaced & p value \\
\hline Pre-operative & 42.7 & 47.5 & $\mathrm{~ns}^{*}$ \\
$1 \mathrm{yr}$ & 61.5 & 68.8 & $\mathrm{~ns}$ \\
$2 \mathrm{yrs}$ & 66.4 & 70.9 & $\mathrm{~ns}$ \\
$4 \mathrm{yrs}$ & 65.8 & 60.5 & $\mathrm{~ns}$ \\
$8 \mathrm{yrs}$ & 62.1 & 51.5 & $\mathrm{~ns}$ \\
$10 \mathrm{yrs}$ & 53.5 & 50.3 & $\mathrm{~ns}$ \\
\hline
\end{tabular}

* ns, not significant

patellar articular surface was fibrillated at the revision procedure but the patella itself was not maltracking. Postoperatively her pain remained unchanged, but she observed a reduction in the click arising from her patella. Her knee score remained unchanged at 95 points.

In the resurfaced group, one patient had a patellofemoral reoperation. This 60-year-old woman had anterior knee pain associated with lateral tilting and underwent an arthroscopic release one year after her primary operation. She subsequently developed a Staphylococcus aureus infection of the knee and multisystem failure. She died of a cerebrovascular accident after a prolonged convalescence. There were no patellar fractures or component loosening seen during the follow-up period.

Outcome scores. Only the pre-operative assessment and the four-year review had a sufficient number of patients to satisfy the power analysis for evaluation using the American Knee Society score. ${ }^{20}$ At the one- and two-year follow-up there were fewer than 37 patients reviewed in one or other treatment group as a result of non-compliance, and these results are not included. Similarly, the loss of patients after four years introduced the likelihood of a type-II error, and the clinical review was discontinued at that stage. The mean clinical score improved from 39.9 points (SD 17.4) preoperatively to 74.9 (SD 14.0) for the non-resurfaced patients and from 36.0 points (SD 14.9) to 71.8 (SD 14.2) for the resurfaced patients at four years. There was no statistically significant difference between the treatment groups. The 50 points allotted to the pain component of the Knee Society score ${ }^{20}$ were analysed as an independent variable and no difference between treatment groups was detected.

The mean Knee Society combining clinical and function scores improved from 86.2 points (SD 28.4) pre-operatively to 135.5 (SD 31.8) for the non-resurfaced patients and from 79.0 points (SD 27.1) to 137.6 (SD 37.7) for the resurfaced patients at four years. The function scores continued to be obtained beyond four years by patient questionnaire and telephone interview; these are given in Table III. The WOMAC ${ }^{22}$ scores were available at eight- and ten-year follow-up (Table IV). The differences were not statistically significant and a type-II error (false negative) may have been present at these intervals because of the reduction in patient numbers. 
Table IV. WOMAC 22 scores $^{*}$

\begin{tabular}{|c|c|c|c|}
\hline & Resurfaced & Not resurfaced & p value \\
\hline \multicolumn{4}{|l|}{ Function } \\
\hline 8 yrs & 35.9 & 36.1 & $\mathrm{~ns}^{\dagger}$ \\
\hline $10 \mathrm{yrs}$ & 31.7 & 37.5 & ns \\
\hline \multicolumn{4}{|l|}{ Pain } \\
\hline $8 \mathrm{yrs}$ & 9.6 & 10.5 & ns \\
\hline $10 \mathrm{yrs}$ & 8.8 & 9.3 & ns \\
\hline \multicolumn{4}{|l|}{ Stiffness } \\
\hline 8 yrs & 3.9 & 4.9 & ns \\
\hline $10 \mathrm{yrs}$ & 3.9 & 4.7 & ns \\
\hline
\end{tabular}

Table V. Patellar specific questions

\begin{tabular}{llll}
\hline & Resurfaced & Not resurfaced & p value \\
\hline Anterior pain (\%) & & & \\
$\quad$ Pre-operative & 52 & 43 & $\mathrm{~ns}^{*}$ \\
4 yrs & 35 & 28 & $\mathrm{~ns}$ \\
8 yrs & 29 & 33 & $\mathrm{~ns}$ \\
10 yrs & 47 & 43 & $\mathrm{~ns}$ \\
& & & \\
Pain with stairs or chairs (\%) & & & \\
$\quad$ Pre-operative & 91 & 88 & $\mathrm{~ns}$ \\
4 yrs & 24 & 29 & $\mathrm{~ns}$ \\
8 yrs & 47 & 51 & $\mathrm{~ns}$ \\
10 yrs & 51 & 69 & \\
& & & \\
Patella crepitus (\%) & & & $\mathrm{ns}$ \\
Pre-operative & 80 & 73 & $\mathrm{~ns}$ \\
4 yrs & 56 & 38 & $\mathrm{~ns}$ \\
8 yrs & 39 & 33 & $\mathrm{~ns}$ \\
10 yrs & 39 & 36 & \\
\hline
\end{tabular}

*ns, not significant

The prevalence of anterior knee pain, function-related pain and patellar crepitus is shown in Table V. We recorded all anterior knee pain including mild pain. There was no statistically significant difference observed between treatment cohorts. The intensity of anterior knee pain, the intensity of pain associated with activity and the intensity of pain in patients with crepitus was analysed using a simple rating scale (none, mild, moderate, severe). No difference in pain intensity was observed using these parameters.

Applying ANOVA with chondromalacia as the grouping variable, no relationship between the grade of chondromalacia of either or both patellar facets was established with pre- or post-operative pain, or anterior knee pain in the resurfaced and non-resurfaced groups. Using chisquared analysis no relationship with pre- or post-operative anterior knee pain was shown.

Radiographic assessment. Patellar tilt and subluxation was significantly different between the two groups. Radiographs of adequate quality were available for 82 patients. Patellar subluxation was absent in all 36 resurfaced patients and was seen in five of 46 non-resurfaced patients, whilst lateral patellar tilt was seen in two resurfaced patients and one non-resurfaced. The incidence of anterior knee pain was not influenced by the presence of patellar tilt or subluxation in either group. In the non-resurfaced group seven of the 46 patients had chondrolysis on the four-year radiographs, but this was not associated with anterior knee pain or activities believed to increase loading on the patella.

The medial retinacular closure markers could be adequately visualised in 64 patients. Separation of the markers by at least $5 \mathrm{~mm}$ was observed in 17 patients. Marker separation was associated with lateral patellar tilt in one nonresurfaced patella, with a wire separation of $7 \mathrm{~mm}$, but was not associated with patellar subluxation or tilt in the remaining 17 patients with radiographic evidence of partial separation of the medial retinacular closure.

There was no change in the position of the joint line by more than $8 \mathrm{~mm}$ in either group. Only one patient had progressive radiolucencies at the tibial interface, but the clinical score was not adversely affected.

\section{Discussion}

This study is one of a small number of randomised prospective trials which have attempted to address the contentious issue of patellar resurfacing in total knee replacement. Similar study designs have been completed in Australia, ${ }^{11}$ North America, ${ }^{13}$ Canada ${ }^{14}$ and the UK. ${ }^{12,16}$ Although the design of the studies was remarkably similar, there were differing philosophies for patellar resurfacing and possibly different patellofemoral demands from the enrolled patients. For example, Australian knee replacement patients tend to live in single-storey dwellings, in contrast to the northern hemisphere where multistorey dwellings and the use of stairs are more common.

Our study has confirmed that total knee replacement results in a significant improvement in function and overall functional capacity in the majority of patients, when assessed using general and specific outcome measures. Our primary statistical assessment to determine whether there would be a difference in the clinical knee scores between the two groups used a $\beta$ (false negative) level of less than 0.05 . If there was a difference between the two groups as defined by our pre-operative goals to determine a clinically relevant change in knee score, it would be achieved within the design parameters of this study. We observed that there was no difference in the knee scores between these two groups, and conclude that our hypothesis that patellar resurfacing in osteoarthritic knees would influence the disease-specific outcome of patients with osteoarthritis undergoing knee replacement was not correct. Three revisions were performed early (less than three years after initial surgery) for patellar problems and there was no additional deterioration in either group which led to further surgery. During the tenyear period of this study we did not observe clinical sequelae from patellar chondrolysis or patellar component failure.

The contemporary literature regarding resurfacing of the patella has conflicting conclusions. Barrack et $\mathrm{al}^{13}$ and Feller et $\mathrm{al}^{6}$ did not demonstrate a significant difference in 
knee score, pain or function when comparing patients with resurfaced or non-resurfaced patellae. Mayman et $\mathrm{al}^{14} \mathrm{dem}-$ onstrated lower rates of anterior knee pain with walking and stair climbing in their resurfaced patients. However, there was no significant difference in Knee Society clinical ratings between the groups. Wood et $\mathrm{al}^{11}$ demonstrated lower rates of anterior knee pain following patellar resurfacing, but no difference in Knee Society score, function score or patient satisfaction.

In contrast, Schroeder-Boersch et $\mathrm{al}^{15}$ and Waters and Bentley $^{12}$ both demonstrated better knee scores but not functional scores in patients with resurfaced patellae. The Swedish Knee Arthroplasty Register reported on 27372 knees with and without resurfacing of the patella, ${ }^{27}$ and suggested that patient satisfaction was greater in resurfaced knees (both in osteoarthritis and rheumatoid arthritis), but over time this difference reduced.

Component design influences patellar tracking and conformity, and may influence the presence of anterior knee pain. Mid-term results from this study echo those from other institutions evaluating the relatively non-conforming Miller-Galante II (Zimmer) implant ${ }^{13}$ and results from the more patellofemoral conforming Anatomic Modular Knee implant (DePuy, Warsaw, Indiana). ${ }^{14}$ Wood et $\mathrm{al}^{11}$ reported a similar randomised study of Australian patients using the Miller-Galante II (Zimmer) implant and reported a low incidence of anterior knee pain in patients with resurfaced patellae. Conversely, a trial from the same institution using a more patella-conforming design reported significantly lower Knee Society scores in the patella-resurfaced patients. ${ }^{10}$ These observations suggest that the results may be design specific.

It has been suggested that patients be stratified to receive patellar resurfacing by the condition of their patellar articular cartilage and the presence of pre-operative anterior knee pain. Boyd et a ${ }^{28}$ recommend selective resurfacing of the patella for patients with degenerative osteoarthritis involving the patella and in those with an inflammatory arthropathy. However, their rate of patellar complications was similar in the two groups, occurring in $4 \%$ of resurfaced and $6 \%$ of non-resurfaced patients. Our study, and that of Barrack et $a l,{ }^{13}$ lacked sufficient patient numbers to isolate the state of the patellar articular cartilage with sufficient statistical sensitivity to make recommendations for selective resurfacing. With our limited numbers we found no significant difference between the presence of pre-operative anterior knee pain and outcome.

Four years after knee replacement, $26(31 \%)$ of our patients exhibited a trend toward more pain and worsening knee function. We included patients with mild anterior knee pain in this assessment. Two patients from the nonresurfaced group had a revision to resurface their patella, with mixed outcome. We have previously reported on the somewhat disappointing results of secondary patellar resurfacing and noted a very high complication rate with this procedure. ${ }^{18}$ If the patella is not resurfaced at the pri- mary replacement, this would appear to expose the patient to a resurfacing risk of between $3 \%$ and $12 \%, 1,13,14,17$ and despite the relatively high complication rate, registry studies suggest that $50 \%$ of these patients will have a satisfactory outcome. ${ }^{27}$ The patella-resurfaced patients had a similar incidence of anterior knee pain. Berry and Rand ${ }^{29}$ had a $39 \%$ major complication rate in their series of isolated revision of the patellar component.

The results of our study and the mid-term results of other randomised prospective controlled trials are unable to provide clear guidelines to recommend routine resurfacing of the patella. Patellar resurfacing remains a philosophical question for the surgeon, who must anticipate that a degree of anterior knee pain will occur in up to a third of patients undergoing knee replacement. ${ }^{1-10}$ Therefore, the decision must be whether to leave the patella unresurfaced and expose the patient to a risk of secondary resurfacing, or alternatively to resurface the patella routinely and accept a similar incidence of anterior knee pain, for which revision options are limited.

The authors gratefully acknowledge the financial support of the Australian Orthopaedic Association and the Adelaide Bone and Joint Research Foundation. We are indebted to the Visiting Orthopaedic Surgeons attending The Queen Elizabeth Hospital whose patients are included in this study, including Mr C. Butcher, P. Dobson, G. Morrison, A. Munyard and P. Lewis.

No benefits in any form have been received or will be received from a commercial party related directly or indirectly to the subject of this article.

\section{References}

1. Ranawat CS. The patellofemoral joint in total condylar knee arthroplasty: pros and cons based on five-to ten-year follow up observations. Clin Orthop 1986;205:93-9.

2. Insall J, Tria AJ, Scott WN. The total condylar knee prosthesis: the first 5 years. Clin Orthop 1979;145:68-77.

3. Freeman MA, Samuelson KM, Elias SG, et al. The patellofemoral joint in total knee prostheses: design considerations. J Arthroplasty 1989;4(Suppl):69-74.

4. Barrack RL, Wolfe MW. Patellar resurfacing in total knee arthroplasty. J Am Acad Orthop Surg 2000;8:75-82.

5. Harwin SF, Stein AJ, Stern RE. Patellofemoral resurfacing in total knee arthroplasty. Contemp Orthop 1994;29:265-71.

6. Feller JA, Bartlett RJ, Lang DM. Patellar resurfacing versus retention in total knee arthroplasty. J Bone Joint Surg [Br] 1996;78-B:226-8.

7. Pollo FE, Jackson RW, Koeter S, et al. Walking, chair rising, and stair climbing after total knee arthroplasty: patellar resurfacing versus nonresurfacing. Am J Knee Surg 2000;13:103-8.

8. Levitsky KA, Harris WJ, McManus J, Scott RD. Total knee arthroplasty without patellar resurfacing: clinical outcomes and long-term follow up evaluation. Clin Orthop 1993;286:116-21.

9. Picetti GD 3rd, McGann WA, Welch RB. The patellofemoral joint after total knee arthroplasty without patellar resurfacing. J Bone Joint Surg [Am]1990;72-A:1379-82.

10. Wood DJ, Smith AJ, Lloyd DG. Clinical outcomes and walking analysis after total knee arthroplasty with and without patella resurfacing: a prospective randomised trial. J Bone Joint Surg [Br] 2005;87-B(Suppl III):338.

11. Wood DJ, Smith AJ, Collopy D, et al. Patellar resurfacing in total knee arthroplasty: a prospective, randomized trial. J Bone Joint Surg [Am] 2002;84-A:187-93.

12. Waters TS, Bentley G. Patellar resurfacing in total knee arthroplasty: a prospective randomized study. J Bone Joint Surg [Am]2003;85-A:212-17.

13. Barrack RL, Bertot AJ, Wolfe MW, et al. Patellar resurfacing in total knee arthroplasty: a prospective, randomized, double-blind study with five to seven years of follow up. J Bone Joint Surg [Am] 2001;83-A:1376-81.

14. Mayman D, Bourne RB, Rorabeck CH, Vas M, Kramer J. Resurfacing versus not resurfacing the patella in total knee arthroplasty: 8- to 10-year results. J Arthroplasty 2003;18:541-45.

15. Schroeder-Boersch H, Scheller G, Fischer J, Jani L. Advantages of patella resurfacing in total knee arthroplasty: two-year results of a prospective randomized study. Arch Orthop Trauma Surg 1998;117:73-8. 
16. Newman JH, Ackroyd CE, Shah NA, Karachalios T. Should the patella be resurfaced during total knee replacement? Knee 2000;7:17-23.

17. Muoneke HE, Khan AM, Giannikas KA, Hagglund E, Dunningham TH. Secondary resurfacing of the patella for persistent anterior knee pain after primary knee arthroplasty. J Bone Joint Surg [Br] 2003;85-B:675-8.

18. Campbell DG, Mintz AD, Stevenson TM. Early patellofemoral revision following total knee arthroplasty. J Arthroplasty 1995;10:287-91

19. Australian Orthopaedic Association National Joint Replacement Registry Annual Report. Adelaide: AOA, 2004.

20. Insall JN, Dorr LD, Scott RD, Scott WN. Rationale of the Knee Society clinical rating system. Clin Orthop 1989;248:13-14.

21. Outerbridge RE. The etiology of chondromalacia patellae. J Bone Joint Surg [Br] 1961;43-B:752-7.

22. Bellamy N, Buchanan WW, Goldsmith CH, Campbell J, Stitt LW. Validation study of WOMAC: a health status instrument for measuring clinically important patient relevant outcomes to antirheumatic drug therapy in patients with osteoarthritis of the hip or knee. J Rheumatology 1988;15:1833-40.
23. Figgie HE 3rd, Goldberg VM, Heiple KG, Moller HS 3rd, Gordon NH. The influence of tibial-patello femoral location on the function of the knee in patients with the posterior stabilized condylar knee prosthesis. J Bone Joint Surg [Am] 1986;68-A 1035-40.

24. Insall JN, Salvati E. Patella position in the normal knee joint. Radiology 1971;101:101-4

25. Gomes LS, Bechtold JE, Gustilo RB. Patella prosthesis positioning in total knee arthroplasty: a roentgenographic study. Clin Orthop 1988;236:72-81.

26. Ewald FC. The Knee Society total knee arthroplasty roentgenographic evaluation and scoring system. Clin Orthop 1989;248:9-12.

27. Robertsson O, Dunbar M, Pehrsson T, Knutson K, Lidgren L. Patient satisfaction after knee arthroplasty: a report on 27,372 knees operated on between 1981 and 1995 in Sweden. Acta Orthop Scand 2000;71:262-7.

28. Boyd AD Jr, Ewald FC, Thomas WH, Poss R, Sledge CB. Long-term complications after total knee arthroplasty with or without resurfacing of the patella. J Bone Joint Surg [Am] 1993;75-A:674-81.

29. Berry DJ, Rand JA. Isolated patellar component revision of total knee arthroplasty. Clin Orthop 1993;286:110-15. 